\title{
MEDIOS DE COMUNICACIÓN DE MASAS Y ESTABLECIMIENTO DE LA AGENDA: UN ESTUDIO EMPÍRICO EN ARGENTINA*
}

\section{Orlando D’Adamo**; Flavia Freidenberg***, Virginia García Beaudoux****}

Fecha de recepción: noviembre 1999

Fecha de aceptación y versión final: abril 2000

\begin{abstract}
Resumen: En el presente artículo se aborda la problemática de los efectos de los medios de comunicación de masas sobre la opinión pública a partir de los resultados obtenidos mediante una investigación empírica. En particular, se analizará la función de establecimiento de la agenda, entendida como la capacidad de los medios de comunicación de dirigir la atención pública hacia ciertas cuestiones particulares. La hipótesis principal propone que los problemas destacados por los medios en su propia agenda temática, terminan siendo los problemas que las personas consideran como los más importantes al elaborar la agenda pública.
\end{abstract}

Palabras clave: Medios de comunicación de masas, efectos psicológicos, establecimiento de la agenda, agenda pública y agenda de los medios.

Abstract: This paper focuses on the mass media effects on public opinion. For that purpose, the results of an empirical research will be used. In particular, we will analyze the agenda setting function, defined as the capacity of the mass media to direct public attention to specific issues. The main hypothesis proposes that the problems the mass media emphasize in their own agen$\mathrm{da}$ are the same problems that people will end to consider as the most important when it comes to elaborate the public agenda.

Key words: Mass Media, Psychological Efects, Agenda Setting, Public Agenda, Mass Media Agenda.

\section{INTRODUCCIÓN}

El papel de los medios de comunicación de masas en la definición de los problemas públicos y en la comunicación de las ideas políticas, ha sido destacado en numerosas ocasiones. En especial, el interés se ha centrado en su poder para guiar la atención pública hacia ciertas controversias y para crear contextos de interpretación de las cuestiones políticas.

Los estudios sobre los efectos de los medios de comunicación de masas comenzaron en las tres primeras décadas del siglo XX. Desde aquel momento hasta la actualidad se pueden distinguir tres grandes paradigmas sucesivos, cada uno de los cuales postuló una particular visión de los efectos que los medios de comunicación producirían sobre las creencias y actitudes de las personas. Desde el modelo hipodérmico de la comunicación de masas (Laswell, 1927) se sostenía que cualquier mensaje presentado adecuadamente produciría efectos instantáneos y masivos sobre las actitudes y comportamientos de la audiencia. En una segunda etapa, iniciada en la década de 1940, esa posición fue abandonada en favor de una postura radicalmente opuesta: la tesis de los efectos mínimos (Klappler, 1974), sustentada sobre el descubrimiento de la selectividad de las funciones cognitivas ${ }^{1}$, que permitió que los investigadores afirmaran que el

* Este trabajo se enmarca en un proyecto más amplio financiado por el Departamento de Investigación de la Universidad de Belgrano (Argentina).

*:* Universidad de Belgrano y Universidad de Buenos Aires (Argentina) $\square$ dagar@pccp.com.ar

**:* Instituto de Estudios de Iberoamérica y Portugal, Universidad de Salamanca (España) y Universidad de Belgrano (Argentina) هlavia@gugu.usal.es

**** Universidad de Belgrano y Universidad de Buenos Aires (Argentina)

1. Como regla general, la selectividad de las funciones cognitivas lo que sugiere es que las personas atienden o se exponen sólo a aquella información que resulta consistente con las creencias y puntos de vista que previamente han formado. La exposición selectiva puede definirse como la tendencia de la audiencia a exponerse a la información más afín a las actitudes que sostiene y a evitar los mensajes que le resulten contrarios a ellas. La percepción selectiva se encuentra emparentada con todos aquellos procesos que inciden en la transformación y modelamiento del significado del mensaje que se ha recibido de un modo acorde con las actitudes y valores de quien lo recibe. Finalmente, la memoria selectiva alude a la tendencia a memorizar aquellos aspectos de los mensajes que resultan coherentes con las propias opiniones y actitudes. 
poder de los medios para cambiar las actitudes de las personas sería mínimo y que, a lo sumo, se limitarían a reforzar las ya existentes. Sin embargo, posterior evidencia sugirió que si bien los procesos selectivos operan en el corto plazo; en el largo plazo, los puntos de vista defendidos por los medios no serían tan inofensivos y podrían dar lugar a efectos más duraderos (Lord, Ross, Lepper, 1979; Kinder, Sears, 1985). En otras palabras, que ejercerían una considerable influencia sobre las opiniones que los individuos manifiestan respecto de las cuestiones de la esfera social. Noelle Neumann (1978) sintetizó el espíritu emergente con relación a los próximos pasos para la investigación que se proponían a partir de esta etapa: “...Después de treinta años en los que el poder de los medios ha sido puesto de continuo en entredicho ... una serie de informes ... dan a entender la necesidad de regresar a la idea de que los medios ejercen una poderosa influencia. No se trata de la noción convencional de influencia ni de la de efectos directos que se propagó en un principio. La tendencia... en el análisis consistiría... en centrarse en la forma sutil e indirecta mediante la que los medios forman nuestra percepción del entorno..." (121). En el marco de este tercer paradigma han surgido, entre otros, los trabajos sobre el fenómeno de fijación o "establecimiento de la agenda", también conocido como "tematización" (McCombs y Shaw, 1968; Iyengar y Kinder, 1987; Katz, 1980).

La Agenda-Setting function, entendida como la capacidad de los medios de comunicación de masas para dirigir la atención pública hacia ciertas cuestiones particulares, postula que los medios de comunicación tendrían la capacidad de decir a los individuos "acerca de qué" se debe pensar $^{2}$, a qué cuestiones se debe prestar atención, con la concomitante desatención de otros eventos que podrían resultar también de interés. El objetivo de este artículo es reflexionar acerca de este proceso a partir de los datos obtenidos de una investigación realizada en Argentina durante el transcurso de 19973.

\section{LA FUNCIÓN DE "ESTABLECIMIENTO DE LA AGENDA"}

A partir de resultados obtenidos en investigaciones relativas a la influencia de las campañas electorales sobre la conducta política, McCombs y Shaw (1972) acuñaron el término "agenda setting" para referirse a una potencialidad de la comunicación de masas: la de dirigir la atención de la opinión pública hacia ciertos temas particulares que los medios de comunicación presentan como los más sobresalientes en un momento determinado. Así, la función del "establecimiento de la agenda" tendría dos consecuencias y características principales. En primer término, los medios sesgan la atención del público hacia determinados objetos o cuestiones de la escena político social; y, en segundo lugar, la asignación de importancia otorgada a cada una de esas cuestiones por los mass media influye sobre el grado de importancia que el público atribuye a esas mismas cuestiones. En palabras de Shaw, "...como consecuencia de la acción de los periódicos, de la televisión y de los demás medios de información, el público es consciente o ignora, presta atención o descuida, enfatiza o pasa por alto, elementos específicos de los escenarios públicos. La gente tiende a incluir o a excluir de sus propios conocimientos lo que los media incluyen o excluyen de su propio contenido. El público además tiende a asignar a lo que incluye una importancia que refleja el énfasis atribuido por los mass media a los acontecimientos, a los problemas, a las personas..." (1979: 96).

Los resultados de los estudios realizados por McCombs y Shaw 4 mostraron la presencia de relaciones entre las percepciones que tienen los electores de la importancia de las cuestiones tratadas en una campaña y el contenido de los mensajes emitidos por los medios. De este modo, la hipótesis que postularon acerca de la función de establecimiento de la agenda, redirigió la mirada de los expertos hacia el poder de los medios y hacia una nueva manera de entender las relaciones entre opinión pública y medios de comunicación de masas. Así, su influencia podría no lograr determinar cómo piensa la gente los diversos fenómenos, pero sería exitosa al delimitar los asuntos en los que se piensa. Esto significa que, además de concentrarse en lo que la gente piensa de las cosas, las investigaciones actuales se interesan por las cuestiones o asuntos "acerca de los que" la gente piensa (Semetko, 1995).

En este sentido, se sostiene que los medios fuerzan la atención hacia determinadas cuestiones, construyen imágenes del mundo político y proponen los objetos acerca de los cuales el público debe pensar. Al describir y precisar la

2. Bernard Cohen (1963) señaló que "Puede ser que la prensa no tenga mucho éxito en indicar a la gente qué pensar, pero tiene un éxito sorprendente a la hora de decir a sus lectores sobre qué pensar ... Diferentes personas tendrán una diferente imagen del mundo en función de ... el mapa que tracen para ellas los escritores, redactores y editores de los periódicos que leen..." (13).

3. Se diseñó un instrumento de investigación que fue administrado en el transcurso de 1997 a una muestra poblacional integrada por 662 estudiantes universitarios argentinos de diferentes orientaciones, con una edad promedio de 21.8 años, siendo el $58.1 \%$ de género femenino y el $41.9 \%$ de género masculino. El instrumento utilizado para relevar los datos consiste en un cuestionario conformado por cuarenta y seis ítems, que fue administrado mediante el método de encuesta. Algunos de los ítems son cerrados, esto es, con opciones múltiples en categorías de respuesta predeterminadas; y otros son abiertos, es decir, dando libertad al encuestado para seleccionar sus respuestas. Contiene además un apartado sociodemográfico que releva edad, género, tipo de carrera que estudia el encuestado, nivel de ingresos familiares, autoubicación ideológica en el continuo "derecha-izquierda" (utilizando para la medición una escala de tipo Likert de 7 gradaciones); y finalmente, una escala de interés por la política.

4. El papel del establecimiento de la agenda fue sometido a prueba empírica en un estudio pionero llevado a cabo en 1968, durante las elecciones presidenciales en Estados Unidos. Se efectuó un sondeo entre los votantes indecisos de Chapel Hill, North Carolina. Encontraron que las cuestiones que los votantes declaraban considerar más importantes coincidían con el grado de cobertura que obtuvieron esas cuestiones en los medios de comunicación locales. El procedimiento consistía en preguntar a los sujetos, mediante un cuestionario, cuales eran los problemas públicos que más los preocupaban y luego se comparaban sus respuestas con categorías derivadas del análisis de contenido de periódicos y programas de televisión. La evidencia indica que los medios ejercen un poderoso impacto sobre las percepciones de los votantes: la correlación entre la importancia que un tema asume en la agenda de los medios y la importancia percibida, es positiva y superior a .9. De 24 pruebas realizadas, 18 favorecieron claramente la hipótesis del establecimiento de la agenda, demostrando que la agenda de los votantes se parecía más a la de los medios de comunicación que, por ejemplo, a la de los partidos políticos. 
realidad externa, los medios dan al público un listado de las cuestiones en torno a las cuales se debe opinar y discutir. Por esa razón, la comprensión de la realidad social que los individuos elaboran es modificada por el accionar de los medios (Shaw, 1979).

Por lo tanto, desde este paradigma, se sustenta que los medios colaborarían en la construcción de actitudes y tendrían la capacidad de orientar selectivamente la atención pública hacia algunos objetos, jerarquizando áreas de problemas sobre los cuales reflexionar. Se trata de la homogeneización de las noticias en un conjunto limitado de temas al que se ciñen la gran mayoría de los medios de comunicación de masas. En otras palabras: los datos de investigaciones ponen de manifiesto correlaciones positivas entre la masiva cobertura periodística de ciertos temas y la posición de fundamental importancia que tales temas ocupan en las jerarquizaciones elaboradas por el público (Funkhouser, 1973; McCombs, 1981).

\section{LA FUNCIÓN DE ESTABLECIMIENTO DE LA AGENDA: EXPLORACIÓN EMPÍRICA}

\section{- Agenda Pública}

La agenda pública se forma a partir de la selección que las personas realizan de los problemas y acontecimientos que consideran como los más relevantes de entre todos los existentes en la escena social. En la investigación se construyó un ítem ${ }^{5}$ con el propósito de relevar la agenda pública, definida como los problemas que la opinión pública considera prioritarios 6 . La intención era comparar dicha agenda con la de los medios de comunicación de masas, definida como los problemas y acontecimientos que los medios de comunicación destacan como los más relevantes, a fin de establecer coincidencias o discrepancias entre ambas.

\begin{tabular}{|c|c|}
\hline \multicolumn{2}{|r|}{ ÜBLICA Y LA AGENDA DE LOS MEDIOS DE COMUNICACIÓN } \\
\hline AGENDA PÜBLICA & $\begin{array}{l}\text { AGENDA DE LOS MEDIOS } \\
{ }^{7}\end{array}$ \\
\hline 1)Desocupación: $70,5 \%$ & 1)Economia/Plan Económico: 25.198 menciones \\
\hline 2)Comupción: $54,2 \%$ & 2) Justicia: 21.836 menciones \\
\hline 3) La crisis en la educación: $38,0 \%$ & 3)Desocupación 11.000 manciones \\
\hline 4) Justicia: $24.6 \%$ & 4) Corrupción: 6.462 menciones \\
\hline 5)Economia: $16,4 \%$ & 5)La crisis en la educ ación: 5.196 menciones \\
\hline 6)Inseguridad: $14,19 \%$ & 6)Inseguridad: 4.336 menciones \\
\hline
\end{tabular}

Como se desprende de los resultados, el público plantea una agenda muy semejante a la fijada por los medios de comunicación. Así, existe una importante convergencia entre lo que el público percibe como importante y lo que los medios caracterizan de ese mismo modo 8 .

\section{- Agenda de los acontecimientos de las 24 horas, 48 horas y 3 semanas}

La hipótesis del establecimiento de la agenda acentúa, desde el punto de vista teórico, la supuesta dependencia cognitiva que los medios generan en los criterios de importancia y organización de los conocimientos que utilizarán los individuos. Los medios no sólo determinan la concienciación sobre el mundo en general a través de los materiales que entregan, sino que también inciden sobre la prominencia de tales elementos (McCombs, 1996). Con respecto a este punto, otro objetivo de la investigación consistió en evaluar si la agenda de los acontecimientos establecida por los encuestados se veía influida por la agenda de los acontecimientos fijada por los medios de comunicación. Así, se procedió a la indagación de cuatro agendas diferenciadas: la de las últimas 24 horas, la de las últimas 48 horas, la de las últimas tres semanas y la de los últimos seis meses 9 . En otras palabras, si los acontecimientos que las personas creen que han sido los más importantes en esos períodos de tiempo, coinciden con los eventos a los que los medios les han dedicado mayor cobertura en esos mismos lapsos.

Analizando los resultados que provienen del análisis de las respuestas de los sujetos encuestados, se encontró que 10:

Con relación a la agenda de las 24 horas, a excepción de un único día, el resto de los días de administración por lo menos un tema de la agen-

5. Item administrado: "Mencione los que Ud. cree que son los tres problemas más importantes del país actualmente”.

6. Existen diversos formatos de ítems para relevar la agenda públi ca, pero ellos guardan gran similitud entre sí. McCombs y Shaw (1972) solicitan a los encuestados que "describan los problemas esenciales, los temas que más les interesan en estos días, con independencia de lo que digan los políticos", y Mullins (1973) que "identifiquen los problemas esenciales que afectan al país" y que indiquen "cuál es el más importante personalmente"

7. En este caso, la "agenda de los medios" fue construida a partir de la suma total de menciones dedicadas a cada tema entre el 13-08-97 y e 20-09-97 en: diario Ámbito Finaciero, Interdiarios, Radio América, Radio Buenos Aires, Radio City, Radio Continental, Radio del Plata, Radio El Mundo, Radio FM 999, Radio FM 999 MDP, Radio FM Cultural, Radio FM Económica, Radio FM La Isla, Radio FM Sol MDP, Radio FM Tango, Radio La Red, Radio Libertad, Radio LT8 Rosario, Radio LU6 Atlántica Radio LU9 MPD, Radio Mitre, Radio Municipal, Radio Rivadavia, Radio Rock \& Pop, Radio Splendid /AM 990, América TV, TV Canal 13, TV Canal 2, TV Canal 9, TV Crónica, TV CV, TV Multicanal, TV Telefé y TV VCC (Fuente: Ámbito Informático CD-ROM).

8. A modo de validación externa, pueden citarse resultados seme jantes a los que hemos obtenido, provenientes de una investigación llevada a cabo por el Centro de Estudios de la Opinión Pública (CEOP) con una muestra representativa de la población de la Capital Federal y el Gran Buenos Aires (CEOP, 1998); así como también datos procedentes del Latinobarómetro para el año 1997. En la agenda pública delineada por nuestros encuestados y en la construida por los sujetos entrevistados por el CEOP, existen innegables coincidencias: desocupación, desempleo, la crisis de la educación, la inseguridad y los problemas económicos son temas destacados en ambas. Algo semejante sucede con los datos presentados en el Latinobarómetro (1997), donde la educación, la corrupción, el desempleo y la justicia aparecen como los problemas más mencionados por los ciudadanos entrevistados.

9. Se pidió a los sujetos que organizaran los acontecimientos y lue go comparamos sus respuestas con la frecuencia de aparición de dichas noticias en distintos medios. Si bien, en general, los estudios indagan a los sujetos en referencia a las últimas 24 o 48 horas y respetamos la medición de esos intervalos de tiempo, nos pareció de interés averiguar si el efecto de establecimiento de la agenda puede resultar más extenso en el tiempo. Esto es, si las personas tendrían la capacidad de construir una agenda para las últimas tres semanas y seis meses, respectivamente. Y, en caso de construir dichas agendas, si en las respuestas se observaría el efecto de "establecimiento de la agenda". De ser así, podría comenzar a plantearse en algún sentido, que el poder de los efectos de los medios es aún más a largo plazo y más profundo de lo que basta el momento se ha propuesto.

10. Para mayores detalles a este respecto sugerimos ver el cuadro anexo. 
da establecida por los encuestados coincide con la agenda pautada por los medios. Y más aún: del total de los días de administración (11 días), el $\mathbf{5 4 , 5} \%$ de ellos (6 días) la agenda de acontecimientos elaborada por los sujetos fue coincidente con la de los medios en más del $50 \%$ de los temas, ascendiendo en algunos casos hasta el $100 \%$ de coincidencia.

- Con relación a la agenda de las 48 horas, en todos los días de administración por lo menos un tema de la agenda elaborada por los encuestados fue coincidente con la agenda establecida por los medios para ese lapso. Del total de los días de administración (11 días) el 72,7\% de ellos (8 días) la agenda de acontecimientos establecida por nuestros entrevistados coincidió con la de los medios al menos en el 50\% de los temas, ascendiendo esa cifra hasta un $75 \%$ de coincidencia.

- Con relación a la agenda de las tres semanas, en la totalidad de los días de administración encontramos que, de mínima, un tema de la agenda de acontecimientos fijada por la muestra coincide con la agenda propuesta por los medios. Del $100 \%$ de los días de administración (11) encontramos coincidencias del 50\% y hasta del 100\%, en el $\mathbf{5 4 , 5 \%}$ de ellos (6 días).

A partir de los datos presentados, se observa una clara tendencia indicativa de que la función de establecimiento de la agenda ha tenido lugar en los tres períodos de tiempo hasta ahora evaluados. Al igual que en otros trabajos de investigación (Stone, 1975; Winter, 1979) se observa en nuestros resultados que las coincidencias en los temas planteados por los sujetos y los medios aumentan a medida que se aproximan al momento de la administración de la encuesta. Un elemento adicional y en común que permitiría explicar la alta coincidencia entre la agenda de los medios y la establecida por los sujetos tanto para las 24 horas, 48 horas, o 3 semanas, radica en que los porcentajes de inclusión de un tema en la agenda de acontecimientos elaborada por los sujetos se vuelven sensiblemente mayores cuando se trata de eventos "espectaculares" o de "Spectacular News Events" (Rogers y Dearing; 1994) 11. Desde el punto de vista del funcionamiento cognitivo, la información más vívida se recuerda mejor. Por lo general, los individuos dedican mayor consideración y otorgan un mayor peso relativo a los datos que son presentados por los medios de manera personal, emocional o acompañados de imágenes, que a los datos que son más válidos desde un punto de vista estadístico pero que resultan emocionalmente neutrales. Los medios de comunicación de masas facilitan ese tipo de procesos al conceder primacía a lo personal frente a lo impersonal: los nombres priman sobre las cosas, el actor sobre el acto (Lapham, 1996). De esta manera, los estímulos y casos vívidos atraen más la atención que los menos vívidos y así resultan ser los que "vienen primero a la mente”, permaneciendo como las categorías más acce- sibles o fácilmente recuperables de la memoria. Esto se refleja, por ejemplo, en los eventos y acontecimientos en los que más del $40 \%$ de la muestra coincidió al momento de elaborar la agenda:

1. Desenlace de la crisis producida por la toma de la Embajada de Japón en Perú: 40,0\%

2. Nuevas Pistas sobre el caso Cabezas 12: $41,1 \%$

3. Muerte de la princesa inglesa Diana Spencer: $43,7 \%$

4. Formación de la Alianza política entre UCRFREPASO 13: 50,98\%

5. Muerte de la Madre Teresa de Calcuta: 55,7\%

6. Polémica y resultados en torno a un control antidoping al que fue sometido Maradona: $82,3 \%$

De este modo, los datos encontrados parecen otorgar sustento tanto a la "hipótesis de la vividez" como a un fenómeno denominado "disponibilidad heurística" 14 o "accesibilidad heurística” (Tversky y Kahneman, 1974). La disponibilidad heurística se define como la tendencia psicológica humana a juzgar un acontecimiento como más probable cuanto más fácilmente pueda ser representado o recordado por la mente.

\section{- Agenda de los seis meses}

Del análisis de la agenda de los 6 meses, en la que se comparó los acontecimientos que los encuestados destacaron como los más importantes de ese lapso con los acontecimientos resaltados por la agenda de los medios para ese mismo período ${ }^{15}$, parece corroborarse nuestra presunción referida a la capacidad de las personas de construir una agenda para un periodo de tiempo más prolongado que el habitualmente investigado.

11. Se define en esas categorías a las noticias que exceden el rango de los temas cotidianamente tratados y que, en muchos casos, adquieren características de show o espectáculo porque permiten tratamientos especiales (por ejemplo, la inclusión de notas con cobertura fotográfica).

12. El "caso Cabezas" se refiere al violento homicidio de un reportero gráfico, hecho que presumiblemente involucra la figura de un empresario íntimamente vinculado con el poder político.

13. La "Alianza UCR-FREPASO" es una alianza electoral creada entre la Unión Cívica Radical y el Frente para un País Solidario.

14. Los heurísticos son reglas informales de pensamiento que están al servicio de simplificar el procesamiento de la información.

15. En este caso, la "agenda de los medios" fue construida a partir de la cantidad de menciones dedicadas a cada tema en: diario Ámbito Finaciero, Interdiarios, Radio América, Radio Buenos Aires, Radio City, Radio Continental, Radio del Plata, Radio El Mundo, Radio FM 999, Radio FM 999 MDP, Radio FM Cultural, Radio FM Económica, Radio FM La Isla, Radio FM Sol MDP, Radio FM Tango, Radio La Red, Radio Libertad, Radio LT8 Rosario, Radio LU6 Atlántica, Radio LU9 MPD, Radio Mitre, Radio Municipal, Radio Rivadavia, Radio Rock \& Pop, Radio Splendid /AM 990, América TV, TV Canal 13, TV Canal 2, TV Canal 9, TV Crónica, TV CV, TV Multicanal, TV Telefé y TV VCC. 


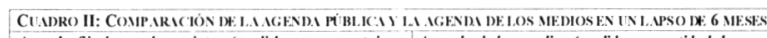
Agenda fijada por los sujetos (metida en porcentajes y. Agenda de los me tios (medida en cuntidlad de mencione.) para el total de la muestrui) 1. Homicidio de José Luis Cab czas: $89,7 \%$ 2. Formación Alian za UCR-FREPASO: $33,8 \%$ 3. La crivin en la cducación: ${ }^{18} 23,+\%$ Segundo juicio por el caso de Maria So kdad Morales: $19,3 \%$ 6. Trere de la Madre Teresa de Calcula: $18.9 \%$ 6. Trasp *o de Hong Kong a China: $11,6 \%$ . Atentados en Medio Oriente: $10,4 \%$ Muerte de la princesa Diana Spencer: $7.8^{\circ} \%$ 1. Homicidio de José Luis Calb cras: 2.216 mencimes 2. Formación Alianza UCR-FREPASO: 805 mencioncs" 3. La crisis en la educación: 795 menciones ${ }^{10}$ 4. Scgundo juicio por el caso de Maria Soledad Moralc: 165 menciones Mescions ${ }^{2}$ 7. Mucrte de la p rincesa Diana Spencer: 54 mezong 23

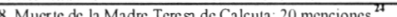

El hecho de que la mayoría de los acontecimientos que las personas priorizaron en la agenda sean coincidentes con los eventos a los que los medios de comunicación dedicaron mayor cantidad de cobertura, podría resultar indicativo de que los efectos de los medios de comunicación de masas sobre la opinión pública se producen aún más a largo plazo y de manera más profunda de lo que se ha postulado hasta el momento en las investigaciones en el área.

En la misma línea de la "hipótesis de la vividez" y su consecuente facilitación de la "disponibilidad heurística" anteriormente comentadas, nos pareció necesario indagar si la agenda de los acontecimientos de los últimos seis meses elaborada por los encuestados sufría alguna modificación luego de dos eventos, muy cercanos en el tiempo, a los que los medios les dedicaron una cobertura extensa y especialmente espectacular. Nos referimos a los fallecimientos de la Madre Teresa de Calcuta (05/08/97) y de la princesa Diana Spencer (31/07/97). La configuración obtenida (en porcentajes en los que cada tema fue incluido en la agenda de los sujetos) fue la siguiente 25 :

1. Homicidio de José Luis Cabezas: $88,6 \%$

2. Muerte de la Madre Teresa: $\mathbf{2 8 , 3} \%$

3. Formación de la Alianza UCR-FREPASO: $25,2 \%$

4. Muerte de la princesa Diana Spencer: $17,8 \%$

5. La crisis en la educación: $13,45 \%$

6. Segundo Juicio por el homicidio de María Soledad Morales: 11,74\%

7. Traspaso de Hong Kong a China: 9,45\%

8. Atentados en Medio Oriente: 7,23\%

Si bien no sería metodológicamente correcto realizar una proyección de estos resultados a la totalidad de la muestra y bajo ningún concepto es nuestra intención hacerlo; creemos, en cambio, que es posible al menos preguntarnos si la configuración de la agenda de los seis meses para el total de la muestra se hubiera visto alterada en el caso de que esos dos eventos hubiesen sucedido al inicio de la administración. En otras palabras, ¿podrían esos dos eventos haber tenido un impacto tal como para modificar la configuración final de la agenda de los 6 meses si en vez de a mediados de la administración hubieran tenido lugar antes de que la misma comenzara, de modo tal que el total de la muestra se hubiera encontrado con la posibilidad de elegirlos?

$\mathrm{Al}$ analizar la agenda de los seis meses, evaluando potenciales elementos en común que podrían compartir los temas incluidos en ella, pensamos en la existencia de una variable que, si bien no ha sido comentada en la literatura acerca del tema, creemos que tendría incidencia y merece

ser tenida en consideración. Nos referimos al poder de influencia que podría tener en la función de establecimiento de la agenda la exposición a grandes cantidades de información acerca de un evento en un breve lapso. A este respecto, una hipótesis adicional que surge a partir de la lectura de los resultados, es la que sugiere que con relación a la información, un impacto constante en una unidad de tiempo breve pero cercana a la evaluación, puede tener más incidencia que el impacto de un evento que se presenta regularmente distribuido en una progresión a lo largo del tiempo.

Analizando los resultados a la luz de los titulares de portada publicados en el diario Clarín (diario leído por el $68,0 \%$ de la muestra), se encuentra que:

* La Alianza UCR-FREPASO fue titular en 15 portadas entre principios de julio y el 20 de septiembre de 1997 (dos meses y medio aproximadamente),

* El segundo juicio para el homicidio de María Soledad Morales, fue titular en $\mathbf{1 2}$ portadas entre mediados de agosto y el 20 de septiembre de 1997 (un mes y medio aproximadamente),

* El fallecimiento de la Madre Teresa de Calcuta, fue titular de portada en 4 oportunidades entre el 5 y el 15 de septiembre de 1997 (diez días),

* El traspaso de Hong Kong a China, obtuvo 7 titulares de portada entre fines de julio y principios de julio (aproximadamente quince días),

* Los Atentados en Medio Oriente, se ubicaron como titular de portada en 9 ocasiones entre fines de julio y el 20 de septiembre de 1997 (un mes y medio aproximadamente),

\section{$\mathrm{y}$}

* El fallecimiento de la princesa Diana Spencer, fue titular en 9 portadas entre el primero y el 20 de septiembre de 1997 (veinte días).

16. Menciones entre el 25/01/97, día del homicidio; y el 20/09/97, último día de la administración.

17. Menciones realizadas entre el $02 / 08 / 97$, día de la creación oficial de la Alianza, y el 20/09/97, último día de la administración

18. Con la denominación "la crisis en la educación" se alude a un profundo conflicto que tuvo lugar entre los docentes de todo el país y el gobierno justicialista.

19. Menciones realizadas entre el $20 / 03 / 97$ y el $20 / 09 / 97$.

20. Menciones realizadas entre el 20/03/97 y el 20/09/97. Si bien el juicio se inició el 15/08/97, desde unos meses antes se comentaba la posibilidad de la apertura de un segundo juicio relativo a este hecho. Se trató del homicidio de una joven oriunda de la provincia argentina de Catamarca que comprometía a la clase política de esta provincia, que puso en evidencia una red de corrupción y encubrimientos, y que conmovió profundamente a la opinión pública de todo el país.

21. Menciones realizadas entre el 01/07/97 (día de la restitución) y el 20/09/97, último día de la administración. Si bien se trató de un período de tiempo breve, el acontecimiento recibió extenso tratamiento

22. Menciones realizadas entre el 31/08/97, día de su fallecimiento; y el 20/09/97, fecha final de la administración

23. Menciones entre el 30/07/97, día del atentado en Israel que desata la ola de violencia; y el 20/09/97, fecha final de la administración

24. Menciones realizadas entre el 05/09/97, día de su fallecimiento; y el 20/09/97, fecha final de la administración.

25. Sujetos encuestados luego del 31/08/97, N=361. 
Así, se puede observar que todos estos acontecimientos destacados por los encuestados comparten la característica de una alta frecuencia de mención en un período breve de tiempo. Se podría especular, entonces, que la exposición a una gran cantidad de información relativa a un tema en un corto tiempo, facilitaría el efecto de recencia 26 , así como también la ya comentada accesibilidad o disponibilidad heurística de esa información. En este caso, la "espectacularidad" del evento, sumada a la frecuente publicación de la noticia en una breve unidad temporal, podrían haber facilitado la disponibilidad de modo tal que el tema quedara accesible, activándose al momento de construir las agendas.

\section{CONCLUSIÓN}

Uno de los principales problemas que ha presentado la hipótesis del establecimiento de la agenda consiste en que los indicadores de la existencia de relaciones entre la cobertura que los medios realizan de cuestiones particulares y la preocupación del público acerca de esas mismas cuestiones, no proveen una evidencia incontestable acerca de los efectos de los medios. Podría ser que la atención que los medios le prestan a determinados problemas simplemente reflejara la preocupación que la opinión pública ya posee respecto de esos problemas. Sin embargo, fue posible efectuar estimaciones más precisas de la dirección causal de dicha relación, de modo de clarificar el panorama. Por sólo citar un caso, en una serie de estudios experimentales, Iyengar y Kinder (1987) editaron telediarios de modo que los sujetos recibieran una dosis constante de noticias acerca de un problema concreto de Estados Unidos. Los sujetos fueron divididos en tres grupos experimentales, en cada uno de los cuales se enfatizó un problema diferente: la debilidad del sistema defensivo de los Estados Unidos, el aumento de la contaminación, la preocupación por la inflación y otras cuestiones económicas. Luego de una semana de exposición a los noticieros editados, los sujetos terminaron convencidos de que el problema más importante que aquejaba a su país era aquel que había recibido más cobertura en el noticiero que les había tocado ver según su grupo experimental. De esa manera, las investigaciones en comunicación encuentran reiteradamente que las historias abordadas por los medios se vinculan con las cuestiones que el público considera las más importantes del momento 27. Los resultados obtenidos a partir de nuestra investigación apuntan en esa misma dirección. Y no sólo se observa el efecto de establecimiento de la agenda en los períodos de 24 horas, 48 horas y tres semanas; sino que también encontramos que dicho efecto puede manifestarse en un lapso más prolongado de lo que las investigaciones han reportado hasta el momento, esto es, de seis meses de duración. Sería interesante en futuras investigaciones explorar la posibilidad de que el efecto de establecimiento de la agenda pudiera tener lugar en períodos aún más extensos de tiempo.

En cuanto a la relación que el efecto de establecimiento de la agenda pudiera tener con otras variables, Iyengar y
Kinder (1987) reportan que cuando alguna persona tiene una experiencia directa con el problema político particular que es el foco de interés de los medios en un determinado momento, se encuentra más expuesta a los efectos del establecimiento de la agenda. Asimismo, su impacto sería mayor en los individuos con menores niveles de educación y de interés por la política. Esto último sugeriría que en una muestra de población general, el impacto debiera ser mayor aún del encontrado entre nuestros estudiantes universitarios.

Más allá del alcance que habitualmente se le atribuye a las teorías del establecimiento de la agenda, que dista de ser limitado, creemos que existe una dimensión que merece ser mencionada y que excede los planteamientos estrictos de estas teorías. Cuando se dice que los medios tendrían la capacidad de establecer temas para la opinión pública, se puede pensar que esa fijación de temas puede constituir un caldo de cultivo favorable a un ulterior apoyo o rechazo de ciertas decisiones políticas o económicas en un determinado conglomerado social. Es decir, grupos políticos o económicos pueden encontrar un ambiente favorable a la concreción de sus objetivos.

Los datos recogidos durante casi treinta años 28 así como los proporcionados por nuestra investigación, indican que los medios tienden a representar el mundo político de manera uniforme y sesgada; y que el público incorpora esos sesgos en los análisis que efectúa de la realidad. Así, la función de establecimiento de la agenda parece constituir el mapa que sirve de guía a la opinión pública en su travesía a través de los espacios públicos y políticos.

26. El efecto de recencia puede ser definido como la mayor facilidad cognitiva para recuperar de la memoria aquella información que se ha incorporado en último lugar.

27. Behr e Iyengar (1985) realizaron mediciones de las mismas variables (inflación, desempleo y energía) a intervalos de tiempo regulares. Encontraron que la influencia causal entre los medios y la opinión pública es unidireccional, esto es, que las preocupaciones de la opinión pública no ejercen influencia sobre las cuestiones que los medios de comunicación de masas eligen cubrir. En un sentido semejante, y como resultado de un estudio experimental, Iyengar, Peters y Kinder (1982) encontraron elementos de corroboración para la hipótesis del establecimiento de la agenda, en otras palabras: que la cobertura de las noticias tiene efecto sobre la importancia percibida de diferentes cuestiones nacionales. En este último caso, los sujetos concurrieron al laboratorio durante una semana, todas las noches, y la tarea consistía en mirar las noticias. Se trataba de un noticiero editado por los investigadores, pero que respetaba el formato habitual de los noticieros televisivos. Cada noche se repetían las historias de la noche anterior, pero con algunas historias agregadas y otras suprimidas. Encontraron que para tres de cada cuatro cuestiones que les presentaron, los rankings de importancia elaborados por los sujetos acerca de una determinada cuestión, aumentaban de manera significativa cuando se trataba de un asunto del que habían visto muchas historias. De este modo, demuestran que las noticias que las cadenas seleccionan como importantes resultan en el aumento correspondiente del lugar de importancia que los espectadores otorgan a esos mismos problemas

28. La recolección de datos en las investigaciones relativas a este tema ha sido efectuada vía procedimientos metodológicos tan disímiles como la evaluación de muestras representativas, las series temporales de estudios comparados basados en muestras representativas, los estudios de panel o los enfoques experimentales. 


\section{ANEXO}

\begin{tabular}{|c|c|c|}
\hline \multicolumn{3}{|c|}{ AGENDAS DE LOS ACONTECIMIENTOS DE LAS 24 HS., 48 HS. Y 3 SEMANAS } \\
\hline $\begin{array}{c}\text { Día de } \\
\text { administración }\end{array}$ & Agenda de los medios & Agenda de los Encuestados \\
\hline 20.08 .97 & $\begin{array}{l}\text { 24 hs.: } \\
\text { 1) ONU-Brasil (pelea por la banca permanente en el Consejo de } \\
\text { Seguridad) } \\
48 \text { hs.: } \\
\text { 1) Constitución de la Alianza UCR-FREPASO } \\
\text { 2) Campaña política del Gobernador Eduardo Duhalde } \\
\text { 3) Denuncias de Domingo Cavallo } \\
\text { 4) ONU-Brasil (pelea por la banca permanente en el Consejo de } \\
\text { 5eguridad) } \\
\text { 5) } 2 .^{\circ} \text { juicio por el homicidio de María Soledad Morales } \\
\text { 3 semanas: } \\
\text { 1) Alianza UCR-FREPASO }\end{array}$ & $\begin{array}{l}24 \text { hs.: } \\
\text { 1) ONU-Brasil (pelea por la banca permanente en el Consejo de } \\
\text { Seguridad): } 66,7 \% \\
48 \text { hs.: } \\
\text { 1) } 2 \text {. }^{\circ} \text { juicio por el homicidio de Maria Soledad Morales: } 32,7 \% \\
\text { 2) ONU-Brasil (pelea por banca } \\
\text { Permanente en el Consejo de Seguridad): } 32,3 \% \\
\text { 3) Proyecto de Reforma Laboral: } 32.3 \% \\
\text { 3 semanas: } \\
\text { 1) Alianza UCR-FREPASO: } 33,3 \% \\
\text { Reactivación del caso del atentado contra la Embajada de Israel en la } \\
\text { Corte Suprema de Justicia: } 33, .3 \% \\
\text { 2) Fassi Lavalle (evasión impositiva): } 16,6 \% \\
\text { Ingreso de la Argentina como socio externo de la OTAN: } 16,6 \% \\
\text { Fuga del Concejal Pico: } 16,6 \%\end{array}$ \\
\hline 25.08 .97 & $\begin{array}{l}\text { 24 hs.: } \\
\text { 1) Diego Maradona vuelve al fútbol (Boca) } \\
\text { 2) Alianza UCR-FREPASO } \\
\text { 3) Exportación de carne argentina a USA } \\
\text { 4) Casos de corrupción en la justicia argentina } \\
\text { 48 hs.: } \\
\text { 1) ONU-Brasil (pelea por la banca permanente en el Consejo de } \\
\text { Seguridad) } \\
\text { 2) 2." juicio por el homicidio de María Soledad Morales } \\
\text { 3 semanas: } \\
\text { 1) Alianza UCR-FREPASO } \\
\text { 2) 2." juicio por el homicidio de María Soledad Morales } \\
\text { 3) Fassi Lavalle (evasión de impuestos) } \\
\text { 4) ONU-Brasil (pelea por la banca permanente en el Consejo de } \\
\text { Seguridad) }\end{array}$ & $\begin{array}{l}24 \text { hs.: } \\
\text { 1) ONU-Brasil (pelea por la banca permanente en el Consejo de } \\
\text { Seguridad): } 32,6 \% \\
\text { 2) Factura de Crédito Dirección General Impositiva: } 18,3 \% \\
\text { Postura contraria de Chile a que Argentina fuera designada como socio } \\
\text { externo de la OTAN: } 18,3 \% \\
\text { 48 hs.: } \\
\text { 1) ONU-Brasil (pelea por la banca permanente en el Consejo de } \\
\text { Seguridad): } 35 \% \\
\text { 2) Campaña política del Gobernador Duhalde: } 18,3 \% \\
\text { Atentados en Medio Oriente: } 18,3 \% \\
\text { 3 semanas: } \\
\text { 1) Alianza UCR-FREPASO: } 33,3 \% \\
\text { ONU-Brasil (pelea por la banca permanente en el Consejo de } \\
\text { Seguridad): } 33,3 \% \\
\text { 2) Paro general: } 16,6 \% \\
\text { 3) 2. } \text { juicio por el homicidio de María Soledad Morales: } 8,3 \% \\
\text { Problemas en el funcionamiento del Mercosur: } 8,3 \% \\
\text { Atentados en Medio Oriente: } 8,3 \% \\
\text { Ingreso de la Argentina como socio externo de la OTAN: } 8,3 \% \\
\text { Proyecto de Reforma laboral: } 8,3 \% \\
\text { Campaña política del Gobernador Duhalde: } 8,3 \%\end{array}$ \\
\hline 26.08 .97 & $\begin{array}{l}24 \text { hs.: } \\
\text { 1) Proyecto de Reforma laboral } \\
\text { 2) Exportación carne argentina a Estados Unidos } \\
\text { 3) Récord de la producción fabril argentina } \\
\text { 4) Casos de corrupción en la justicia argentina } \\
\text { 5) Fassi Lavalle (evasión impositiva) } \\
\text { 48 hs.: } \\
\text { 1) ONU-Brasil (pelea por la banca permanente en el Consejo de } \\
\text { 2) Deguridad) } \\
\text { 3) Exportación carne argentina a Estados Unidos } \\
\text { 4) Alianza UCR-FREPASO } \\
\text { 5) Casos de corrupción en la justicia argentina } \\
\text { 3 semanas: } \\
\text { 1) Alianza UCR-FREPASO } \\
\text { 2) ONU-Brasil (pelea por la banca permanente en el Consejo de } \\
\text { Seguridad) } \\
\text { 3) 2. juicio por el homicidio de María Soledad Morales }\end{array}$ & $\begin{array}{l}24 \text { hs.: } \\
\text { 1) Exportación carne argentina a Estados Unidos: } \mathbf{1 9} \% \\
\text { 2) Proyecto de Reforma laboral: } \mathbf{1 6} \% \\
\text { Campaña política del Gobernador Duhalde: } 16 \% \\
\text { 3) Investigación por el homicidio de J.L. Cabezas (Nuevos datos tras las } \\
\text { declaraciones de Ríos): } 15 \% \\
\text { Alianza UCR-FREPASO: } 15 \% \\
\text { 48 hs.: } \\
\text { 1) ONU-Brasil (pelea por la banca permanente en el Consejo de } \\
\text { Seguridad): } 28 \% \\
\text { 2) Alianza UCR-FREPASO: } 21 \% \\
\text { 3) 2." juicio por el homicidio de María Soledad Morales: } 15 \% \\
\text { 4) Ingreso de la Argentina como socio externo de la OTAN: } 10 \% \\
\text { 5) Exportación carne argentina a Estados Unidos: } 9 \% \\
\text { 3 semanas: } \\
\text { 1) Alianza UCR-FREPASO: } 33 \% \\
\text { 2) ONU-Brasil (pelea por la banca permanente en el Consejo de } \\
\text { Seguridad): } 22 \% \\
\text { 3) 2. juicio por el homicidio de María Soledad Morales: } \mathbf{1 4} \%\end{array}$ \\
\hline 27.08 .97 & $\begin{array}{l}24 \text { hs. } \\
\text { 1) Ajuste tarifas telefónicas } \\
\text { 2) } 2 .^{\circ} \text { juicio por el homicidio de María Soledad Morales } \\
\text { 3) Privatización del Correo argentino } \\
\text { 4) Fassi Lavalle (evasión de impuestos) } \\
\text { 5) Casos de corrupción en la justicia argentina }\end{array}$ & $\begin{array}{l}24 \text { hs.: } \\
\text { 1) Privatización del Correo argentino: } 25 \% \\
\text { 2) } 2 .^{\circ} \text { juicio por el homicidio de María Soledad Morales: } 10 \% \\
\text { 3) Alianza UCR-FREPASO: } 5 \% \\
\text { Exportación carne argentina a Estados Unidos : } 5 \% \\
\text { Ajuste de las tarifas telefónicas: } 5 \%\end{array}$ \\
\hline
\end{tabular}




\begin{tabular}{|c|c|c|}
\hline & $\begin{array}{l}48 \text { hs.: } \\
\text { 1) Diego Maradona vuelve a jugar al fútbol (Boca) } \\
\text { 2) Alianza UCR-FREPASO } \\
\text { 3) Exportación carne argentina a Estados Unidos } \\
\text { 4) Casos de corrupción en la justicia argentina } \\
\text { 5) Proyecto de Reforma laboral } \\
\text { 6) Récord producción fabril argentina } \\
\text { 7) Fassi Lavalle (evasión impositiva) } \\
\text { 3 semanas: } \\
\text { 1) Alianza UCR-FREPASO } \\
\text { 2) ONU-Brasil (pelea por la banca permanente en el Consejo de } \\
\text { 2) } 2 \text {. juicio por el homicidio de María Soledad }\end{array}$ & $\begin{array}{l}48 \text { hs.: } \\
\text { 1) Estado de situación referida a la Toma de los Rehenes en la Embajadal } \\
\text { de Japón en Perú: } 40 \% \\
\text { 2) Privatización del Correo argentino: } 35 \% \\
\text { 3) Alianza UCR-FREPASO: } 10 \% \\
\text { 2. Juicio por el homicidio de María Soledad Morales: } 10 \% \\
\text { Envío de una sonda a Marte: } 10 \% \\
\text { 3 semanas: } \\
\text { 1) Alianza UCR-FREPASO: } 30 \% \\
\text { 2) Visita del Papa a París y encuentro con la juventud: } 20 \% \\
\text { 3) Fassi-Lavalle (evasión impositiva):15\% } \\
\text { 4) 2. juicio por el homicidio de María Soledad Morales : } \mathbf{1 0} \% \\
\text { Diego Maradona vuelve a jugar al fútbol (Boca): } 10 \% \\
\text { Atentados en Medio Oriente: } 10 \%\end{array}$ \\
\hline 28.08 .97 & $\begin{array}{l}24 \text { hs.: } \\
\text { 1) Privatización de los aeropuertos } \\
\text { 2) Fassi Lavalle (evasión impositiva) } \\
\text { 3) Renuncia de Martín Redrado } \\
\text { 4) Doping Maradona } \\
\text { 5) 2. }{ }^{\circ} \text { juicio por el homicidio de María Soledad Morales } \\
\text { 48 hs.: } \\
\text { 1) Proyecto de Reforma laboral } \\
\text { 2) Exportación carne argentina a Estados Unidos } \\
\text { 3) Récord producción fabril argentina } \\
\text { 4) Casos de corrupción en la justicia argentina (Mafia judicial) } \\
\text { 5) Fassi Lavalle (evasión impositiva) } \\
\text { 6) Ajuste de las tarifas telefónicas } \\
\text { 7) 2. juicio por el homicidio de María Soledad Morales } \\
\text { 8) Privatización del Correo argentino } \\
\text { 3 semanas: } \\
\text { 1) Alianza UCR-FREPASO } \\
\text { 2) ONU-Brasil (pelea por la banca permanente en el Consejo de } \\
\text { 3) } 2 \text {. }^{\circ} \text { juicio por el homicidio de María Soledad Morales }\end{array}$ & 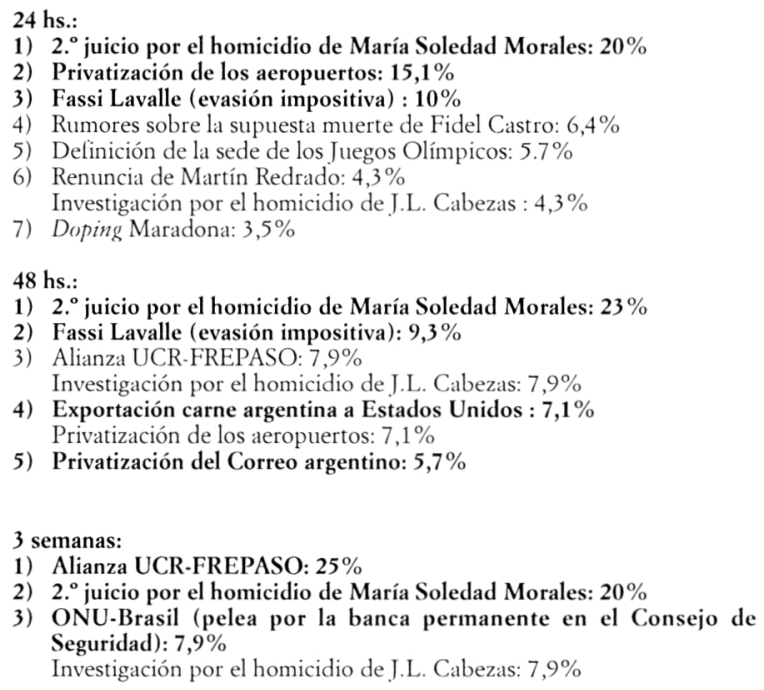 \\
\hline 29.08 .97 & $\begin{array}{l}\text { 24 hs.: } \\
\text { 1) Campaña política del Gobernador Duhalde } \\
\text { 2) Doping Maradona } \\
\text { 3) Aniversario del atentado a la AMIA (aparición de nuevas pistas } \\
\text { para la resolución del caso) } \\
\text { 4) } 2 .^{\circ} \text { juicio por el homicidio de María Soledad Morales } \\
\text { 5) Investigación por el homicidio de J.L.Cabezas } \\
48 \text { hs.: } \\
\text { 1) Ajuste de las tarilas telefónicas } \\
\text { 2) } 2 .^{\circ} \text { juicio por el homicidio de María Soledad Morales } \\
\text { 3) Privatización del Correo argentino } \\
\text { 4) Fassi Lavalle (evasión impositiva) } \\
\text { 5) Casos de corrupción en la justicia argentina (Mafia judicial) } \\
\text { 6) Privatización de los aeropuertos } \\
\text { 7) Renuncia de Martín Redrado } \\
\text { 8) Doping Maradona } \\
\text { 3 semanas: } \\
\text { 1) Alianza UCR-FREPASO } \\
\text { 2) ONU.Brasil (pelea por una banca permanente en el Consejo de } \\
\text { 3) Seguridad) } \\
\text { 2. juicio por el homicidio de María Soledad Morales }\end{array}$ & $\begin{array}{l}24 \text { hs.: } \\
\text { 1) Doping Maradona: } 82,3 \% \\
\text { 2) Investigación por el homicidio de J.L.Cabezas: } 41,1 \% \\
\text { 3) } 2{ }^{\circ} \text { juicio por el homicidio de María Soledad Morales: } 21,5 \% \\
\text { 4) Campaña política del Gobernador Duhalde: } 17,6 \% \\
48 \text { hs.: } \\
\text { 1) } 2 .{ }^{\circ} \text { juicio por el homicidio de Maria Soledad Morales: } 37.2 \% \\
\text { 2) Fassi Lavalle (evasión impositiva): } 27,4 \% \\
\text { 3) Alianza UCR-FREPASO: } 11,7 \% \\
\text { 4) ONU-Brasil (pelea por la banca permanente en el Consejo de } \\
\text { Seguridad): } 5,8 \% \\
\text { Doping Maradona: } 5.8 \% \\
\text { Exportación de carne argentina a Estados Unidos: } 5,8 \% \\
\text { Investigación por el homicidio de J.L.Cabezas: } 5,8 \% \\
\text { Rumores sobre la supuesta muerte de Fidel Castro: } 5,8 \% \\
\text { 3 semanas: } \\
\text { 1) Alianza UCR-FREPASO: } 50,98 \% \\
\text { 2) 2. juicio por el homicidio de María Soledad Morales: } 45 \% \\
\text { 3) ONU-Brasil (pelea por una banca permanente en el Consejo de } \\
\text { Seguridad): } 25,4 \% \\
\text { 4) Fassi Lavalle (evasión de impuestos): } 17,6 \% \\
\text { 5) Exportación de carne argentina a Estados Unidos: } 11,7 \% \\
\text { 6) Investigación por el homicidio de J.L.Cabezas: } 9,8 \%\end{array}$ \\
\hline 12.09 .97 & $\begin{array}{l}24 \text { hs.: } \\
\text { 1) Aumento de salarios a los docentes } \\
\text { 2) Reunión de los presidentes de los países miembros del Mercosur } \\
\text { 3) Actividades en memoria del expresidente Salvador Allende en } \\
\text { Chile } \\
48 \text { hs.: } \\
\text { 1) Denuncias de Domingo Cavallo } \\
\text { 2) Doping Maradona } \\
\text { 3) La Selección Argentina clasificó para jugar el Mundial de Fútbol } \\
\text { 4) 2. juicio por el homicidio de María Soledad Morales } \\
\text { 5) Renuncias en el gobierno } \\
\text { 3 semanas: } \\
\text { 1) Doping Maradona } \\
\text { 2) Investigaciones por el homicidio de J.L.Cabezas } \\
\text { 3) 2. juicio por el homicidio de María Soledad Morales } \\
\text { 4) Fallecimiento de La princesa Diana Spencer } \\
\text { 5) Fuga del Concejal Pico } \\
\text { 6) Fallecimiento de la Madre Teresa de Calcuta }\end{array}$ & 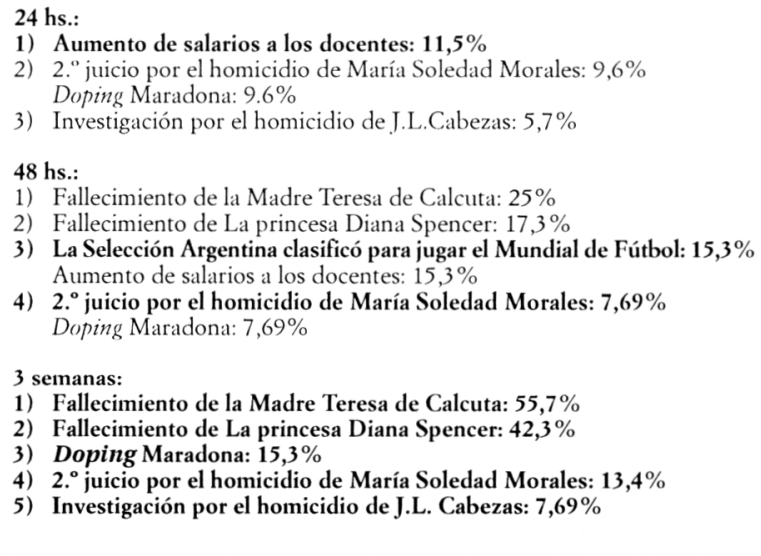 \\
\hline
\end{tabular}




\begin{tabular}{|c|c|c|}
\hline 15.09 .97 & $\begin{array}{l}24 \text { hs.: } \\
\text { 1) Diego Maradona juega otra vez al fútbol (Boca) } \\
\text { 2) Aumento en el gasto público destinado a los jubilados y a los } \\
\text { docentes } \\
\text { 3) Abogados-sobornos } \\
48 \text { hs.: } \\
\text { 1) Aumento de los salarios a los docentes } \\
\text { 2) Disminuyen las muertes a causa del SIDA } \\
\text { 3) Fallecimiento de la Madre Teresa de Calcuta } \\
\text { 3 semanas: } \\
\text { 1) Doping Maradona } \\
\text { 2) Investigación por el homicidio de J.L.Cabezas } \\
\text { 3) 2. juicio por el homicidio de María Soledad Morales } \\
\text { 4) Fallecimiento de La princesa Diana Spencer } \\
\text { 5) Fallecimiento de la Madre Teresa de Calcuta }\end{array}$ & 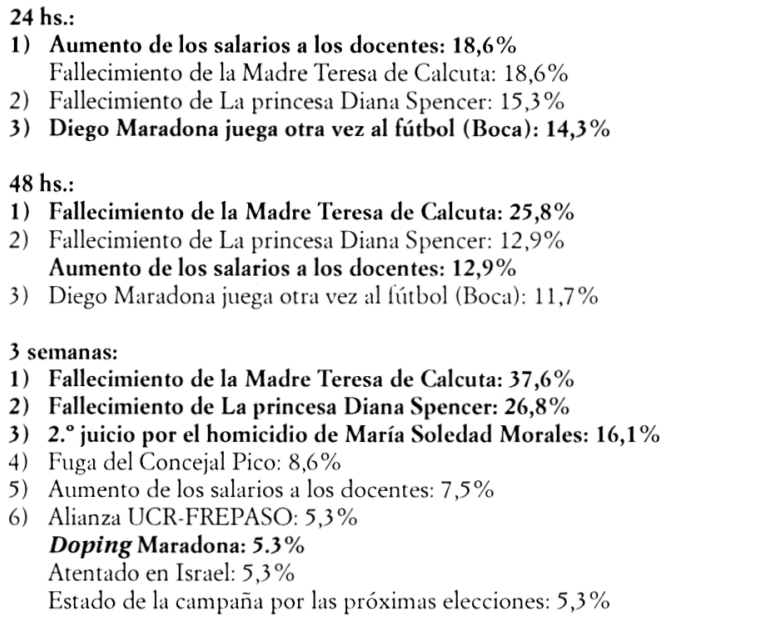 \\
\hline 17.09 .97 & $\begin{array}{l}24 \text { hs.: } \\
\text { 1) Moratoria en los plazos de la Dirección General Impositiva } \\
\text { 2) Aumento en las pensiones de los jubilados } \\
\text { 3) Disminuyen las inuerte a causa del SIDA } \\
\text { 4) Diego Maradona vuelve a jugar al fútbol (Boca) } \\
\text { 5) Descubren al un médico que ejercía sin título } \\
\text { 6) Procesan al ex intendente Carlos Grosso } \\
\text { 7) Subsidios parra jefes de tamilia desempleados } \\
\text { 8) Abogados-sobornos } \\
\text { 48 hs.: } \\
\text { 1) Diego Maradona vuelve a jugar al fútbol (Boca) } \\
\text { 2) Aumentos en las pensiones de los jubilados } \\
\text { 3) Abogados-sobornos } \\
\text { 4) Disminuyen las muertes a causa del SIDA } \\
\text { 5) 2." Doping a Maradona } \\
\text { 6) Disturbios en las manifestaciones en recuerdo de la "Noche de los } \\
\text { Lápices" } \\
\text { 3 semanas: } \\
\text { 1) } 1 .{ }^{\circ} \text { Doping Maradona } \\
\text { 2) Investigaciones por el homicidio de J.L.Cabezas } \\
\text { 3) Fallecimiento de La princesa Diana Spencer } \\
\text { 4) Fallecimiento de la Madre Teresa }\end{array}$ & $\begin{array}{l}24 \text { hs.: } \\
\text { 1) Diego Maradona vuelve a jugar al fútbol (Boca): } \mathbf{1 8 , 9 2} \% \\
\text { 2) Disturbios en las manifestaciones de recuerdo por la "Noche de los } \\
\text { Lápices": } 18,0 \% \\
\text { 3) Aumento en las pensiones de los jubilados: } 17,1 \% \\
\text { 4) Fallecimiento de La princesa Diana Spencer: } 12,67 \% \\
\text { Fallecimiento de la Madre Teresa de Calcuta: } 12,67 \% \\
\text { Aumentos en los salarios a los docentes: } 12,67 \% \\
\text { Disminuyen las muertes a causa del SIDA: 12,67\% } \\
\text { 48 hs.: } \\
\text { 1) Aumentos en las pensiones de los jubilados: } 23,2 \% \\
\text { 2) Aumentos en los salarios a los docentes: } 12,9 \% \\
\text { 3) Fallecimiento de la Madre Teresa de Calcuta: } 11,6 \% \\
\text { 4) Diego Maradona vuelve a jugar al fútbol (Boca): 8,0\% } \\
\text { 5) Disturbios en las manifestaciones en recuerdo de la "Noche de los } \\
\text { Lápices": 7,1\% } \\
\text { 2." juicio por el homicidio de María Soledad Morales: 7,1\% } \\
\text { Disminución de las muertes a causa del SIDA: 7,1\% } \\
\text { 6) Fallecimiento de La princesa Diana Spencer: 6,25\% } \\
\text { Abogados-sobornos: 6,25\% } \\
\text { 7) 2." Doping Maradona: } 5,3 \% \\
\text { 3 semanas: } \\
\text { 1) Fallecimiento de la Madre Teresa de Calcuta: } 54,4 \% \\
\text { 2) Fallecimiento de La princesa Diana Spencer: } 43,7 \% \\
\text { 3) 2." juicio por el homicidio de María Soledad Morales: } 17,8 \% \\
\text { 4) Doping Maradona: } 16,9 \% \\
\text { 5) Investigaciones por el homicidio de J.L.Cabezas: } 8,0 \%\end{array}$ \\
\hline 18.09 .97 & $\begin{array}{l}24 \text { hs.: } \\
\text { 1) Aumento en las pensiones de los jubilados } \\
\text { 2) 2. juicio por el homicidio de María Soledad Morales } \\
\text { 3) Doping Maradona } \\
\text { 4) Moratoria en los plazos de la Dirección General Impositival } \\
\text { 5) La Alianza gana en la provincia del Chaco } \\
\text { 6) Abogados-sobornos } \\
\text { 7) Ecología y Cataratas } \\
\text { 48 hs.: } \\
\text { 1) Aumento en las pensiones de los jubilados } \\
\text { 2) Disminuyen las muertes a causia del SIDA } \\
\text { 3) Diego Maradona vuelve a jugar al fútbol (Boca) } \\
\text { 4) Doping Maradona } \\
\text { 5) Disturbios en las manifestaciones en recuerdo de la "Noche de } \\
\text { los Lápices" } \\
\text { 6) Abogados-sobornos } \\
\text { 7) Moratoria Dirección General Impositiva } \\
\text { 8) Descubren a un médico que ejercía sin título } \\
\text { 9) Procesan al ex intendente Carlos Grosso } \\
\text { 10) Subsidios a los jeles de familia desempleados } \\
\text { 3 semanas: } \\
\text { 1) Doping Maradona } \\
\text { 2) Investigaciones por el homicidio de J.L.Cabezas } \\
\text { 3) Fallecimiento de La princesa Diana Spencer } \\
\text { 4) Fallecimiento de la Madre Teresa de Calcuta }\end{array}$ & 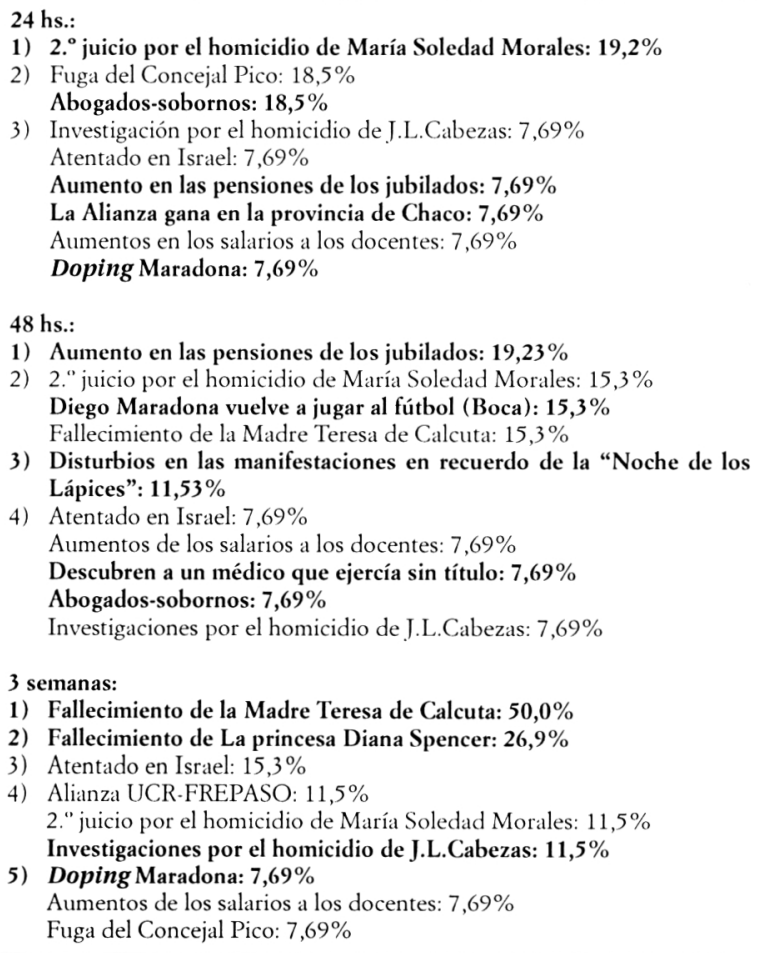 \\
\hline
\end{tabular}




\begin{tabular}{|l|l}
20.09 .97 & 24 hs.: \\
1) Declaraciones de Carlos Menem contra la prensa (Interpretación \\
de la Ley del Palo de Benjamin Franklin) \\
2) Bicipolicías en la Capital Federal \\
3) Diego Maradona vuelve a jugar al fútbol (Boca) \\
4) Descubren a un médico que ejercía sin título \\
48 hs.: \\
1) 2. juicio por el homicidio de María Soledad Morales \\
2) Declaraciones de Carlos Menem contra la prensa (Interpretación \\
3) Ee la Ley de Palo de Benjamín Franklin) \\
4) Matan a turistas en Egipto \\
5) Aumento en las pensiones de los jubilados \\
6) Doping Diego Maradona \\
7) Moratoria en los plazos de la Dirección General Impositiva \\
8) Alianza UCR-FREPASO \\
9) Abogados-sobornos \\
10) Descubren a un médico que ejercía sin licencia \\
3 semanas: \\
1) Doping Maradona \\
2) Investigaciones por el homicidio de J.L. Cabezas \\
3) Fallecimiento de La princesa Diana Spencer \\
4) Fallecimiento de la Madre Teresa de Calcuta
\end{tabular}

24 hs.:

1) Diego Maradona vuelve a jugar al fútbol (Boca): $10,25 \%$

2) Aumento en las pensiones de los jubilados: $7,69 \%$ Declaraciones de Carlos Menem contra la prensa (Interpretación de la Ley de Palo de Benjamín Franklin) : 7,69\%

Matan a turistas en Egipto: 7,69\%

3) 2. juicio por el homicidio de María Soledad Morales: $5,1 \%$ Fallecimiento de la Madre Teresa de Calcuta: 5,1\%

48 hs.

1) 2. juicio por el homicidio de María Soledad Morales: $10,25 \%$

2) Doping Diego Maradona: 7,69\%

Fallecimiento de La princesa Diana Spencer: 7,69\% Aumento en las pensiones de los jubilados: $7,69 \%$ Declaraciones de Carlos Menem contra la prensa (Interpretación de la Ley de Palo de Benjamín Franklin): 7,69\%

Estado de la campaña por las próximas elecciones: 7,69\%

3) Investigaciones por el homicidio de J.L. Cabezas: $5,1 \%$ Atentado en Israel: $5,1 \%$

Fallecimiento de la Madre Teresa de Calcuta: 5,1\%

\section{3 semanas:}

1) Fallecimiento de la Madre Teresa de Calcula: $43,5 \%$

2) Fallecimiento de La princesa Diana Spencer: $35,89 \%$

3 Exportación carne argentina a Estados Unidos: 12,8\% Estado de la campaña por las próximas elecciones: $12,8 \%$

4) Investigaciones por el homicidio de J. L. Cabezas: 7,69\% 2. ${ }^{\circ}$ juicio por el homicidio de María Soledad Morales: 7,69\%

El texto resaltado en negrita señala los temas que han resultado coincidentes entre la agenda de los entrevistados y la agenda de los medios de comunicación. Elaboración de los autores

\section{REFERENCIAS BIBLIOGRÁFICAS}

Ámbito Informático CDROM (1998). Buenos Aires: Diario Ámbito Financiero.

BEHR, R.; Iyengar, S. (1985): "Television news, real-world cues, and changes in the public agenda". En Public Opinion Quarterly, 49, 38-57.

CEOP (1998): ver: Diario Clarín, Domingo 27 de septiembre de 1998, suplemento de Política, p. 4.

Cohen , B. (1963): The press and foreign policy. Princeton: Princeton University Press.

FunKhouser, G. (1973): "The issues of the sixties: An exploratory study in the dynamics of public opinion". En Public Opinion Quarterly, 37, 62-75.

IyenGAR, S. y D. Kinder (1987): News that matters: AgendaSetting and priming in a television age. Chicago: University of Chicago Press.

KATZ, E. (1980): "On conceptualizing media effects". En: T. MACCORMAK (comp.): Communications studies: decade of dissent. Greenwich, Conn: JAI Press.

Kinder, D. Y D. SEARS (1985): "Public opinion and political action”. En: LINDZEY, y E. ARONSON (comps.): The Handbook of Social Psychology, vol. II. New York: Random House.

Klapper, J. (1974): Efectos de la comunicación de masas. Madrid: Aguilar.

LAPHAM, L. (1996): “El ahora eterno”. Prólogo a la edición de la MIT Press del año 1994 de: M. McLuHAN: Comprender los medios de comunicación. Las extensiones del ser humano. Ed. Consultada: 1996. Barcelona: Paidós.

Lasswell, H. (1927): Propaganda Technique in the World War. New York: Alfred A. Knopf.

LATINOBARÓMETRO (1997). Madrid: Corporación Latinobarómetro.

LORD, C.; Ross, L. y M. Lepper (1979): "Biased asimilation and attitude polarization: The effects of prior theories on subsequently considered evidence". En Journal of Personality and Social Psychology, 37, 2098-2109.
McCombS, M. (1981): “The agenda-setting approach”. En: D. Nimmo y K. SANDERS (comps.): Handbook of political communication. California: Sage.

MCCOMBS, M. (1996): "Influencia de las noticias sobre nuestras imágenes del mundo”. En: J. BRYANT y D. ZillmanN (comps.): Los efectos de los medios de comunicación. Barcelona: Paidós.

McCOMBS, M.; Shaw, D. (1972): "The agenda-setting function of mass media”, Public Opinion Quarterly, 36, 176-187.

Mullins, L. (1973): Agenda-setting on the campus: the mass media and learning of issues importance in the 72 Election. Colorado: Association for Education in Journalism.

Noelle-Neumann, E. (1978): L'influence des mass media. Allensbach: Etudes de radio-television.

Rogers, E.; DeAring, J. (1994): "Agenda-Setting Research: Where has it been, where is it going?”. En: D. GRABER (comp.): Media Power in Politics. Washington D.C.: Congressional Quarterly.

SemetKo, H. (1995): "Investigación sobre tendencias de la agenda-setting de los noventa”. En: A. MuÑOZ-AlOnSO, J. Rospir (comps.): Comunicación Politica. Mdrid: Ed. Universitas

SHAW, E. (1977): "The interpersonal agenda". En: D. Shaw, E. McCombs (comps.): The emergence of American Public Issues: The agenda-setting function of the press. St. Paul, Mn: West.

SHAW, E. (1979): "Agenda-setting and mass communication theory”, Gazette (International Journal for Mass Communication Studies), vol XXV, n. ${ }^{\circ}$ 2, 96-105.

STONE, G. (1975): "Cumulative effects of the media". Trabajo presentado en la Conferencia sobre Agenda-Setting de la Universidad de Syracuse.

TVERSKY, A.; Kahneman, D. (1981): "The framing of decisions and the psychology of choice", Science, 211, 453-58.

WINTER, J. (1979): "An agenda-setting frame for the civil rights issue, 1954-1976”. Trabajo realizado en la Universidad de Syracuse. 